\title{
La formación del profesor de Educación Física en las Necesidades Educativas Especiales, hacia la reflexión y la acción, desde acuerdos internacionales y Leyes en Chile The training of the Physical Education teacher in Special Educational Needs, towards reflection and action, from international agreements and Laws in Chile
}

\author{
Antonio Castillo-Paredes \\ Universidad de Las Américas (Chile)
}

Resumen. El Informe Warnock y la Declaración de Salamanca, establecieron criterios estandarizados sobre conceptos y requerimientos mínimos para una enseñanza homogénea de los centros educativos para las personas con Necesidades Educativas Especiales (NEE). Las NEE dentro de la normativa en Chile, se clasifican en Necesidades Educativas Especiales Permanentes y Transitorias. Las cuales, mediante decretos, leyes y acuerdos internacionales, los niños, niñas y adolescentes tienen el derecho de una educación de calidad, teniendo en consideración las características particulares que pueden presentar los estudiantes, este acompañamiento puede ser transitorio y/o permanente. De esta manera, se busca que, a través de la Educación Especial, el alumnado obtenga herramientas necesarias para su utilización en contextos sociales y laborales. Sin embargo, desde la Educación Física en contextos escolares, se evidencia poca preparación o conocimiento de las características de las NEE que presente un estudiante por parte del profesor de Educación Física, si bien, en distintas universidades se encuentra la asignatura que dote a los estudiantes de pedagogía de herramientas y competencias para el trabajo con escolares con NEE, se evidencia que existe una falta de componentes teóricos y prácticos para trabajar con NEE. Finalmente, se invita a la reflexión para la utilización de actividades físico - deportivas o motrices adaptadas, las cuales podrían permitir ser una herramienta complementaria e inclusiva en la clase de Educación Física escolar.

Palabras claves: Necesidades educativas especiales; Educación Física; Adaptaciones curriculares; Escuela; Universidad; Persona en situación de discapacidad.

Abstract. The Warnock Report and the Salamanca Declaration established standardized criteria on concepts and minimum requirements for a homogeneous teaching of educational centers for people with Special Educational Needs (SEN). SEN within the regulations in Chile are classified as Permanent and Transitory Special Educational Needs. Which, through decrees, laws and international agreements, children and adolescents have the right to a quality education, taking into consideration the characteristics that students may present, this accompaniment may be transitory and / or permanent. In this way, it is sought that, through Special Education, students obtain the necessary tools for their use in social and work contexts. However, from Physical Education in school contexts, little preparation, or knowledge of the characteristics of SEN presented by a student is evidenced by the Physical Education teacher, although, in different universities there is the subject that provides students pedagogy of tools and competencies for working with schoolchildren with SEN, it is evident that there is a lack of theoretical and practical components to work with SEN. Finally, reflection is invited for the use of adapted physical - sports or motor activities, which could allow them to be a complementary and inclusive tool in the school Physical Education class.

Keywords: Special educational needs; Physical education; Curriculum adaptations; School; University; Disabled persons.

\section{Introducción}

El informe de Warnock fue creado por el Comité de Investigación sobre la Educación de Niños y Jóvenes Deficientes bajo el título Special Educational Needs a petición de la Secretaría de Educación del Reino Unido en 1978 (Warnock, 1987). Este documento, buscó considerar sugerencias conceptuales sobre las Necesidades Educativas Especiales (NEE) relacionados con la búsqueda de una enseñanza homogénea, considerando as-

Fecha recepción: 21-09-21. Fecha de aceptación: 19-12-21

Antonio Castillo-Paredes

acastillop85@gmail.com pectos de los centros educativos, evaluativos, integración física, social y funcional (Aguilar, 1991). Así mismo, en la Declaración de Salamanca y UNESCO en la Conferencia Mundial de 1994, se afirmó que «la integración y la participación son parte esencial de la dignidad humana y del goce y ejercicio de los derechos humanos» (UNICEF, 2005. p13).

Las Necesidades Educativas Especiales (NEE) tienen relación con «aquellos estudiantes, que, sin importar la causa, presentan dificultades para el aprendizaje y requieren recursos adicionales de manera momentánea o continua» (UNICEF, 2005.p71). De esta manera, en Chile, el Decreto Exento $N^{\circ} 83 / 2015$ conceptualiza las NEE en Necesidades educativas especiales de carác- 
ter permanente: visual, auditiva, disfasia, trastorno autista, discapacidad intelectual y discapacidad múltiple, y en NEE de carácter transitorio: asociadas a las dificultades del aprendizaje, trastornos específicos del lenguaje (TEL), déficit atencional y coeficiente intelectual limítrofe (MINEDUC, 2015).

De esta manera, se espera que el contexto educativo en Chile busque establecer una armonía con el Informe Warnock y la Declaración de Salamanca reflejado en el Decreto No 87, Ley 20422 y Ley 20845. Sin embargo, desde la perspectiva docente la integración escolar resulta compleja o se desvía de su objetivo si no se generan las circunstancias para el progreso hacia la inclusión (Tenorio, 2005), debido a que debe existir un equilibrio en las responsabilidades a nivel social, comunitario, educativa y familiar (Soto, 2003).

La participación de la familia, escuela y comunidad son fundamentales para el logro de la inclusión, dado que en primera instancia la familia es la base de la realidad social, desde la organización, funcionamiento y estructura a la cual obedece, considerando la convivencia cotidiana, moral y ética (Calvo, Verdugo \& Amor, 2016; Simón, Giné \& Echeita, 2016; Villalobos, 2015), desde la comunidad o sociedad, la cual debe facilitar la interacción social establecida desde las políticas de gobierno relacionadas con la accesibilidad a la salud, educación y el trabajo (Juárez, Comboni \& Garnique, 2010) y desde la escuela permite dar respuesta desde la inclusión educacional, participación social y aceptación a la diversidad (Molina, 2015), pero, desde la Educación Física escolar existe la necesidad de mejorar la formación sobre las NEE de manera teórica y práctica en la formación de profesores de Educación Física (Campos, Llopis, Gimeno \& Maher, 2020), debido a que según las percepciones del alumnado con NEE señalan que los profesionales de Educación Física no se encuentran preparados para trabajar con ellos (Vila et al., 2021). Con base a lo anteriormente señalado, el presente artículo tiene por objetivo reflexionar sobre la formación profesional del profesor de Educación Física en las NEE en Chile.

\section{Contexto}

La presente investigación presenta el acontecer en Chile relacionado con la Educación Física y las NEE. De esta manera, bajo la legislación chilena en torno a leyes, decretos, documentos ministeriales y acuerdos internacionales relacionadas con el Informe Warnock, la Declaración de Salamanca, entre otros. El presente traba- jo permitirá al lector, reflexionar sobre la legislación y normativa en Chile, la formación del profesorado y el uso de las actividades físico-deportivas o educativas como herramienta complementaria a la clase de Educación Física y Salud. Permitiendo de esta manera realizar la transferencia de los beneficios educativos, sociales, biológicos, motrices y psicológicos en niños y adolescentes con (Necesidades Educativas Especiales Permanentes) y NEET (Necesidades Educativas Especiales Transitorias), concibiendo de esta manera la obtención de herramientas y habilidades para el desarrollo de un adulto íntegro para la sociedad en la cual viven.

\section{Acuerdos Internacionales y Legislación en Chi- le}

Para la integración e inclusión de las NEE a nivel nacional e internacional a nivel social y educativo se encuentran el Informe Warnock (Warnock, 1987) y la Declaración de Salamanca (UNICEF, 2005. p13), los cuales establecen lineamientos para la consideración de la formación de niños, niñas y adolescentes con NEE como seres íntegros para la sociedad, traduciéndose en un equilibrio biopsicosocial. En el caso de Chile, en contextos educativos, las políticas públicas establecen Decretos que permiten la integración educativa (MINEDUC, 2015), beneficios de subvenciones para la educación especial (MINEDUC, 2009) e instructivos que norman la Educación Especial (MINEDUC, 2021), la cual tuvo sus inicios en 1852 (Caiceo, 2011).

Las Bases Curriculares, mediante el Decreto 433 (BCN, 2012) establece en el artículo $3^{\circ}$ que la Educación Física y Salud constituye una asignatura esencial en la educación escolar, las cuales destacan la importancia del movimiento, desarrollo de la condición física, cualidades expresivas, iniciación deportiva, conjunción de factores para una vida activa, seguir las reglas del juego, cooperación y trabajo en equipo y liderazgo en la enseñanza básica de $1^{\circ}$ a $6^{\circ}$ básico. En $7^{\circ}$ y $8^{\circ}$ básico, $1^{\circ}$ y $2^{\circ}$ enseñanza media el Decreto Ley 369 (BCN, 2015) la Educación Física y Salud se encuentra orientada a la promoción del bienestar y autocuidado. En $3^{\circ}$ y $4^{\circ}$ de enseñanza media a través del Decreto 193 (BCN, 2019) la Educación Física y Salud busca el desarrollo de conocimientos, habilidades y actitudes que permitan la práctica regular de actividad física, calidad de vida, incentivo de espacios públicos, transformación de promotores de una vida activa y saludable en contextos escolares y sociales, mediante la aplicación y liderazgo a través de programas y proyectos deportivos, recreativo y socioculturales. 
Sin embargo, González (1998) señaló que las Bases Curriculares en Chile no proponen adecuaciones curriculares (objetivos, contenidos, metodología, evaluación, temporalización) y provisión de medios de acceso al currículum (situaciones educativas especiales, recursos personales, recursos materiales, acceso físico y dependencias de los establecimientos escolares). Además, considerando lo mencionado por González (1998), luego de 23 años después, Castillo-Retamal et al. (2021a) reafirman que las Bases Curriculares de Educación Física y Salud en Chile, no poseen lineamientos claros para el trabajo con estudiantes con NEE, debido a que solo se considera de manera más objetiva el desarrollo de los objetivos para estudiantes con desarrollo típico y no están diseñadas para la inclusión. Esto, tampoco permite considerar la duración de una clase, traduciéndose en un posible estrés y cansancio emocional de los escolares con NEEP (Campos, 2021).

El acceso a la Educación Especial se encuentra respaldada en la Ley 20845 (BCN, 2015), en el cual dentro de su artículo $1^{\circ}$ señala que «Es deber del Estado permitir el aseguramiento a la educación inclusiva de calidad para todas las personas y las condiciones necesarias para el acceso y permanencia de los estudiantes NEE en establecimientos de educación regular o especial». De esta manera, el Decreto 83 establece criterios y orientaciones para la adecuación curricular en la Educación Parvularia y Educación Básica (MINEDUC, 2015), las cuales están en concordancia con el artículo 23 de la Ley 20370 en relación la Educación Especial o Diferencial que se desarrolla de manera transversal, dotando a los estudiantes con NEE de servicios, recursos humanos, técnicos y conocimientos especializados de manera permanente o transitoria y en según la Ley 20422 donde en su párrafo $2^{\circ}$ contiene 9 artículos relativos a la educación e inclusión escolar. De esta manera la creación de escuelas inclusivas permite eliminar las barreras del aprendizaje y promueve la participación de los estudiantes (López, Julio, Morales \& Pérez, 2014). Sin embargo, a pesar de la legislación en Chile, se requiere la creación de una política educativa integral, la cual debe permitir un cambio real y significativo en la inclusión educativa del país (Iturra, 2019).

\section{Formación del Profesor de Educación Física y NEE}

Un proceso de reflexión lleva consigo aspectos de razonamiento pedagógico relacionados con la revisión, reconstrucción, representación y análisis crítico de un desempeño o acción propiamente tal. Bajo esta lógica, para concebir un proceso de reflexión le anteceden aspectos relacionados con la comprensión, transformación, enseñanza y evaluación, de esta manera se gestan nuevas formas de comprender y aprender nuevas experiencias (Shulman, 2005). Este proceso de reflexión nos permite llevar a cabo transformaciones educativas y curriculares para la implementación de los planes y programas de manera flexible en el contexto escolar, así se podrán adaptar y organizar de manera coherente ante la realidad educativa existente (Osandón et al., 2018), permitiendo el desarrollo del currículum oculto y subyacente, instaurando flexibilidad curricular para el desarrollo de la enseñanza del alumnado (Casero, García \& Moratilla, 2012). Sin dejar de lado que el currículo educativo de un país fundamenta la proyección visionaria de las competencias que requiere un ciudadano para su integración social (Duk \& Loren, 2010).

La Educación Física en el sistema escolar en Chile, se rige bajo la normativa ministerial a través de la asignatura de «Educación Física y Salud». De esta manera, se da respuesta a sus orientaciones que se sustentan en la práctica regular de actividad física, permitiendo el desarrollo de las habilidades motrices, en los establecimientos educativos a lo largo del país, además el escolar debe adquirir actitudes inclinadas hacia el juego limpio, liderazgo y autocuidado, para la obtención de beneficios personales y sociales, como la mantención de estilos de vida activos y saludables (UCE, 2021).

Sin embargo, para dar cumplimiento a las leyes y normativas vigentes hacia contexto escolar, la Ley 20903 crea el sistema de desarrollo profesional docente (BNC, 2016) y esta formación de docentes, a través del Centro de Perfeccionamiento, Experimentación e Investigaciones Pedagógicas (CPEIP) del Ministerio de Educación (MINEDUC) declara que la Calidad Docente implica el fortalecimiento durante la formación de futuros profesores y profesoras, mediante el apoyo y acompañamiento en los primeros años del ejercicio de la profesión docente (CPEIP, 2020). De esta manera, el ejercicio de la profesión docente es guiado por el «Marco referencial para la Calidad Docente», el cual describe, a través de los Estándares orientadores para carreras de pedagogía en Educación Física estándares disciplinares y pedagógicos (CPEIP, 2018) hacia el contexto escolar. Desde los «Estándares disciplinares de Educación Física y Salud», se busca la formación integral del escolar, de manera «motriz, afectivo, social, cognitivo, moral y espiritual» (MINEDUC, 2014, p21.), los cuales integran las Necesidades Educativas Especiales Transitorias (NEET) y Permanentes (NEEP). 
Con base en lo anterior, Flores \& Maureira (2020) señalaron que, de las 26 mallas de Pedagogía en Educación Física analizadas de universidades en Chile, solo siete de ellas no consideran asignaturas relacionadas con la inclusión en la formación inicial de profesores de Pedagogía en Educación Física, y recientemente Muñoz (2021a) señaló que luego de la evaluación de 27 planes de estudios, el 3,88\% de las asignaturas se encuentran ligadas a la inclusión, traduciéndose en un 5,04\% de horas del total de la carrera. Sin embargo, los estudiantes de Pedagogía en Educación Física, durante su proceso de práctica profesional (virtual) indicaron que, si bien los centros de prácticas atienden a sus escolares con NEE, algunos de ellos señalaron que existe una baja preparación recibida sobre las NEE e inclusión (Castillo-Retamal et al., 2021b).

Tomando en consideración lo anteriormente señalado, esto se traduce a que las Escuelas especiales en Chile, deberían permitir el desarrollo de un ser íntegro para la sociedad en la cual se desenvuelve. Pero en la actualidad no existen programas que fomenten la promoción del bienestar y la salud física - funcional, además que permitan la transferencia de las herramientas, competencias y habilidades adquiridas hacia el área laboral (Farías et al., 2021). De esta manera se presenta como un reto para las universidades en Chile en la construcción de las mallas hacia la inclusión y la discapacidad tomando como referencia la práctica de actividad física adaptada (Muñoz, 2021b), también, se podría considerar la motricidad adaptada y deportes adaptados permitiendo ser una herramienta complementaria de la formación de profesores y profesoras en la inclusión (Miranda \& Médor, 2018).

\section{Conclusiones}

En referencia al Informe Warnock, han pasado alrededor de 43 años desde su publicación, desde esa fecha, 16 años más tarde la Declaración de Salamanca en 1994 representó un compromiso de los países y de la sociedad hacia las personas con NEE. Sin embargo, la literatura analizada aún señala que la sociedad aún carece de compromiso social, educativo y laboral hacia las NEE.

La normativa y la legislación en Chile establecen herramientas, directrices y ordenanzas para la integración e inclusión de las NEE en la sociedad, pero, la realidad educativa, social y familiar no se encuentran en armonía, lo cual no permiten la transferencia de saberes, conocimientos, competencias y herramientas para el desempeño en la sociedad en la cual se desenvuelve.
Desde la Formación Inicial Docente del profesor de Educación Física, los documentos ministeriales no profundizan de manera sólida las consideraciones para el trabajo con NEE, repercutiendo en las mallas que forman docentes, lo cual se traduce en una discordancia entre el ejercicio de la profesión docente, las orientaciones técnicas y las leyes vigentes para la profundización curricular de la Educación Física y Salud hacia las NEE.

De esta manera, se invita hacia una reflexión pedagógica, la cual permite la integración de recursos personales cognitivos, metacognitivos y emocionales (Correa, Chaubet, Collin \& Gervais, 2014), las cuales podrán permitir enfrentar, organizar y replantear aspectos teóricos - prácticos dentro de la formación inicial docente, documentos orientadores, legislativos hacia el ejercicio de la profesión desde una realidad educativa para el logro de aprendizajes significativos de los estudiantes con NEE desde la Educación Física en contexto escolar.

\section{Aplicaciones prácticas}

Con base en la legislación en Chile, que establece las definiciones y características de las NEET y las NEEP (López \& Valenzuela, 2015), en primera instancia es necesario conocer las características particulares de los estudiantes para realizar las adecuaciones pertinentes al currículum. La literatura científica evidencia intervenciones que pueden ser utilizadas como herramientas complementarias a la clase de Educación Física. Para el caso de NEET, en niños y adolescentes con TDAH intervenciones desarrolladas a través de actividades físico - deportivas permiten mejoras de la sintomatología de este trastorno en la inatención e hiperactividad (Palma et al., 2021). En el caso de NEEP, el desarrollo de actividad física o ejercicio físico en niños y adolescentes con trastorno del espectro autista permite la mejora del desempeño motor (Montalva-Valenzuela, OlivaresArancibia \& Castillo-Paredes, 2021).

También, se ha evidenciado intervenciones complementarias como la equinoterapia para personas con NEE y/o discapacidad para ser utilizada como una opción terapéutica (Jiménez, Jaime \& Figueredo, 2019). A su vez, el uso de modelos físico - educativos a través de estaciones motrices físico - educativas para potenciar habilidades y capacidades motrices para estudiantes con NEE auditivas, físicas, psicosociales y visuales (Bonifaz et al., 2018). Siguiendo este razonamiento, el desarrollo de programas de Educación Deportiva y Aprendiza-Servicio por parte de los profesores de Educación Física, per- 
mitiría el desarrollo de habilidades sociales y personales en el alumnado con NEE (Abellán, Segovia, Gutiérrez \& García, 2021).

Estas intervenciones complementarias a la clase de Educación Física, con el apoyo de actividades psicoeducativas y socioemocionales (Anita \&Widyarini, 2020; Pegalajar, 2017), deberán tener una duración de $45 \mathrm{~min}$ a $75 \mathrm{~min}$ de trabajo, realizados dos o tres veces a la semana, siempre teniendo en consideración a las características personales de los estudiantes con NEET o NEEP (Montalva-Valenzuela, Olivares-Arancibia \& Castillo-Paredes, 2021; Ochoa-Martínez et al., 2018; Palma et al., 2021). Además de considerar la formación permanente del profesor de Educación Física, para facilitar transformaciones de manera positiva en el sistema escolar (González \& Macías, 2017).

\section{Futuras líneas de investigación}

Si bien, las Bases Curriculares (UCE, 2021) no establecen directrices de manera concreta para profundizar en la inclusión, se considera pertinente que, mediante la práctica regular de actividad física, los educandos pueden desarrollar habilidades motrices, sociales, trabajo colaborativo, respeto, autocuidado y liderazgo. De esta manera, podrían otorgar «la posibilidad de adquirir un estilo de vida activo y saludable, asociado a múltiples beneficios individuales y sociales» en niños y adolescentes con NEE, permitiéndoles la obtención de herramientas y habilidades transferibles a la adultez.

De esta manera, nuevas investigaciones a través de intervenciones de actividades físico-deportivas, permitirán evidenciar los beneficios de la práctica regular de actividad física a nivel educativo, social, emocional y motriz. Además, de esta manera, podrían emerger nuevas estrategias metodológicas, didácticas y complementarias para la clase de Educación Física en contextos escolares.

\section{Referencias}

Abellán, J., Segovia,Y., Gutiérrez,D., \& García,L. M. (2021). Sensibilización hacia la discapacidad a través de un programa integrado de Educación Deportiva y Aprendizaje-Servicio (Disability awareness through an integrated program of sport education and service-learning). Retos, 43, 477-487. https://doi.org/10.47197/ retos.v43i0.86625

Aguilar, L. (1991). El informe warnock. Cuadernos de pedagogía, 197, 62-64. https://blocs.xtec.cat/ educacioespecialssdh / files / 2009/01/informewarnock.PDF
Anita, L., \&Widyarini, N. (2020). PsychoeducationTraining Intervention for Emotional Intelligence in Special School X. Social Sciences \& Psychology , 65(1), 11-11.https:// doi.org/10.47119/IJRP1006511120201560

BCN (2016). Decreto 169 Exento, Modifica Plan de Estudio de Séptimo a Segundo año de Enseñanza Media y Programas que Indica. https://www.bcn.cl/leychile/ navegar?i=1060269

BCN (2019). Decreto 193, Aprueba Bases Curriculares para los Cursos de $3^{\circ}$ y $4^{\circ}$ año de Educación Media, en asignaturas que indica. https://www.bcn.cl/leychile/ navegar?idNorma $=1136078$

BCN (2016). Decreto 369, Establece Bases Curriculares desde $7^{\circ}$ año Básico a $2^{\circ}$ año Medio, en Asignaturas que Indica.https: / / www.bcn.cl/leychile / navegar $\mathrm{i}=1084868 \& \mathrm{f}=2016-08-06 \& \mathrm{p}=$

BCN (2012). Decreto 433, Establece Bases Curriculares para laEducación Básica en Asignaturas que Indica.https: / /www.bcn.cl/leychile/navegar?idNorma=1047359

BCN (2010). Ley 20370, Establece la Ley General de Educación.https: / / www.bcn.cl/leychile/ navegar?idNorma $=1006043$

BCN (2021). Ley 20422, Establece Normas Sobre Igualdad De Oportunidades E Inclusión Social De Personas Con Discapacidad. https://www.bcn.cl/leychile/ navegar?idLey $=20422$

BCN (2015). Ley 20845, De Inclusión Escolar que Regula la Admisión de Los y Las Estudiantes, Elimina el Financiamiento Compartido y Prohibe el Lucro en Establecimientos Educacionales que reciben aporte del Estado. https://www.bcn.cl/leychile/ navegar?idNorma $=1078172$

BCN (2016). Ley 20.903, Sistema de Desarrollo Profesional Docente. Recuperado en: https: / /www.bcn.cl/ leychile/navegar?idNorma $=1087343$

Bonifaz, I., Chávez, J., Trujillo, H., Sánchez, J., Palacios, D., \& Álvarez, E. (2018). Estaciones motrices físico-educativas para niños y adolescentes con necesidades asociadas o no a discapacidades. Revista Cubana de Investigaciones Biomédicas, 37(4), 1-13. http://scielo.sld.cu/ scielo.php?script $=$ sci_arttext $\&$ pid $=$ S0 864 03002018000400012

Caiceo, J. (2011). Esbozo de la educación especial en Chile: 1850-1980. Revista Educación y Pedagogía, 22(57), 31-49. https:/ / revistas.udea.edu.co/index.php/revistaeyp/ article/view/9839

Calvo, M., Verdugo, M. Á., \& Amor, A. M. (2016). La participación familiar es un requisito imprescindible para una escuela inclusiva. Revista latinoamericana de educación inclusiva, 10(1), 99-113. http://dx.doi.org/10.4067/ S0718-73782016000100006 
Campos, K. (2021). Afectividad de un niño con trastornos del espectro autista en el contexto escolar y familiar: estudio de un caso. Journal of Movement \& Health, 18(1). https: / / doi.org/ 10.5027 /jmh-Vol18Issue1(2021)art105

Campos, J., Llopis, R., Gimeno, M., \& Maher, A. (2020). Percepción de competencia para la atención de alumnos con necesidades educativas especiales en Educación Física: la voz de estudiantes universitarios de España y Reino Unido (Perceived competence to teach students with special educational needs in Physic. Retos, 39, 372-378. https: / / doi.org/10.47197/retos.v0i39.79498

Casero, J., García, A. I., \& Moratilla, A. (2012). Análisis del currículum oculto en el área de conocimiento del medio en algunos libros de tercer ciclo de primaria. Revista Ibero-Americana de Estudos em Educação, 7(3), 15-24. https: / /doi.org/10.21723/riaee.v7i3.5629

Castillo- Retamal, F. Cárcamo, B., Aravena, H., Valenzuela, A., Pérez,T., Medel, C., \& Quezada, J. (2021). Necesidades Educativas Especiales y Educación Física: un análisis desde la propuesta curricular ministerial de Chile. Retos: nuevas tendencias en educación física, deporte y recreación, (42), 56-65. https:/ / doi.org/10.47197/ retos.v42i0.86977

Castillo-Retamal, F., Torres-Medina, A., Herrera-Muñoz, M., \& Faúndez-Casanova, C. (2021). Percepciones sobre el abordaje de las Necesidades Educativas Especiales en la Práctica Profesional Virtual de Educación Física (Perceptions on addressing Special Educational Needs in the virtual professional practice of Physical Education). Retos, 42, 774-784. https://doi.org/10.47197/ retos.v42i0.87310

Correa, E., Chaubet, P., Collin, S., \& Gervais, C. (2014). Desafíos metodológicos para el estudio de la relexión en contexto deformación docente. Estudios Pedagógicos, 40(1), 71-86. http://dx.doi.org/10.4067/S071807052014000200005

CPEIP. (2020). Que es Calidad Docente. https:// www.cpeip.cl/que-es-calidad-docente/

CPEIP (2018). Estándares orientadores para carreras de pedagogía en educación física. https: / / www.cpeip.cl/ w p - content/ u ploads / $2018 / 09 /$ Est\%C3\%A1ndares_Educacion_fisica-2.pdf

Duk, C. \& Loren, C. (2010). Flexibilización del currículum para atender la diversidad. Revista Latinoamericana de Educación Inclusiva, 4(1), pp. 187-210. http:// www.rinace.net/rlei/numeros/vol4-num1/art9.pdf.

Farías, C., Ferrari, G., Espoz-Lazo, S., Jofré-Saldía, E., Ferrero, P., \& Valdivia-Moral, P. (2021). Escuelas especiales de Chile: $;$ Responsables del desarrollo de la condición física-funcional para la inclusión laboral de perso- nas con discapacidad intelectual?. Journal of Movement \& Health, 18(2). https: / /doi.org/10.5027/jmh-Vol18Issue2(2021)art109

Flores, E., \& Maureira, F. (2020). Formación pedagógica en la carrera de educación física: Falta de conocimientos para un profesional del siglo XXI. EmásF: revista digital de educación física, (62), 118-126. https:// dialnet.unirioja.es/servlet/articulo?codigo $=7186184$

Jimenez, R., Jaime, L., \& Figueredo, L. (2019). La monta terapéutica como Actividad Física Adaptada (AFA) para personas con Necesidades Educativas Especiales (NEE)(Original). Olimpia: Publicación científica de la facultad de cultura fisica de la Universidad de Granma, 16(54), 206-215. https://dialnet.unirioja.es/servlet/ articulo?codigo $=7000705$

Juárez, J. M., Comboni, S., \& Garnique, F. (2010). De la educación especial a la educación inclusiva.Argumentos (México, DF), 23(62), 41-83. http: / /www.scielo.org.mx/ scielo.php?script $=$ sci_arttext\&pid $=$ S0 187 57952010000100003

González, M. (1998). Necesidades educativas especiales/ generales hacia una nueva denominación. Revista GalegoPortuguesa de Psicoloxía e Educación, 2 (3). https: / / core.ac.uk/download/pdf/61899886.pdf

González, I., \& Macías, D. (2017). La formación permanente como herramienta para mejorar la intervención del maestro de educación física con alumnado con discapacidad (Lifelong learning as a tool to improve physical education teachers' intervention with students with disabilities). Retos, 33, 118-122. https: / /doi.org/ 10.47197/retos.v0i33.55056

Iturra, P. (2019). Dilemas de la inclusión educativa en el Chile actual. Revista Educación Las Américas, 8, 80-92. https: / /doi.org/10.35811/rea.v8i0.7

López, V., Julio, C., Morales, M., Rojas, C., \& Pérez, M. V. (2014). Barreras culturales para la inclusión: políticas y prácticas de integración en Chile. Ministerio de Educación. Revista de educación, 363 (11). https:// www.educacionyfp.gob.es/revista-de-educacion/ numeros-revista-educacion/numeros-anteriores/2014/ 363/re363-11.html

López, S. I. M., \& Valenzuela, B. G. E. (2015). Niños y adolescentes con necesidades educativas especiales. Revista Médica Clínica Las Condes, 26(1), 42-51. https: / / doi.org/ 10.1016/j.rmclc.2015.02.004

Miranda, M.A., \& Médor, P. R. (2018). Formación inicial de un profesorado inclusivo: avances y perspectivas en la carrera de pedagogía en educación física, deporte y recreación de la Universidad Austral de Chile. Paideia Surcolombiana, (23), 106-121. https://doi.org/ 10.25054/01240307.1843 
Molina, Y. (2015). Necesidades educativas especiales, elementos para una propuesta de inclusión educativa a través de la investigación acción participativa: El caso de la Escuela México. Estudios pedagógicos (Valdivia), 41(ESPECIAL), 147-167. http://dx.doi.org/10.4067/S071807052015000300010

MINEDUC (2015). Diversificación de la enseñanza. Decreto $\mathrm{N}^{\circ} 83 / 2015$ Aprueba Criterios y Orientaciones de Adecuación Curricular para Estudiantes con Necesidades Educativas Especiales de Educación Parvularia y Educación Básica. https: / / especial.mineduc.cl/wpcontent/uploads / sites/31/2016/08/Decreto-832015.pdf

MINEDUC (2009). Decreto $\mathrm{N}^{\circ} 170$. Fija normas para determinar los alumnos con necesidades educativas especiales que serán beneficiarios de las subvenciones para la educación especial. https:// especial.mineduc.cl/wpcontent/uploads/sites/31/2016/08/ 201304231500550.DEC200900170.pdf

MINEDUC (2021). Decretos e instructivos vigentes que normal la Educación Especial. https:// especial.mineduc.cl/normativa/decretos-e-instructivos/

MINEDUC (2014). Estándares orientadores para carreras de Pedagogía en Reducación Física. Recuperado en: https: / / www.cpeip.cl/wp-content/uploads/2019/03/ Est \%C3\%A1ndares_Educacion_fisica-2.pdf

Muñoz, F. (2021a). Formación del profesorado de educación física vinculado a la inclusión y la discapacidad en universidades de Chile. Journal of Movement \& Health, 19(1). http: / / dx.doi.org/10.5027/jmh-Vol19Issue1(2022)art134

Muñoz, F. (2021b). Discapacidad e Inclusión, un desafío para la Educación Física en Chile. Journal of Movement \& Health, 18(1). https: / /doi.org/10.5027/jmh-Vol18Issue1(2021)art98

Ochoa-Martínez, P., Hall López, J., Carmona López, A., Reyes Castro, Z., Sáenz-López Buñuel, P., \& Conde García, C. (2018). Análisis comparativo de un programa educación física en niños con discapacidad auditiva sobre la edad motora equivalente (Comparative analysis of the effect of physical education program of motor age equivalent in children with hearing disability). Retos, 35 , 310-313. https: / /doi.org/10.47197/retos.v0i35.67190

Osandón, L., Caro, M., Magendzo, A., Lavín, S., González, F., \& Cabaluz, J. (2018). Estado, mercado y currículum escolar: La experiencia chilena (1964-2018); Cuestiones fundamentales y actuales del currículo, el aprendizaje y la evaluación. https://unesdoc.unesco.org/ark:/48223/ pf0000265296

Palma,T., Carroza, D. , Torres, R., Poblete-Aro, C., Cadagan,
C., \& Castillo-Paredes, A. (2021). Cambios en los síntomas de inatención, hiperactividad e impulsividad en niños y adolescentes con TDAH mediante los Deportes. Una revision (Changes in the symptoms of inattention, hyperactivity and impulsivity in children and adolescents with ADHD through. Retos, 41, 701-707. https:/ / doi.org/10.47197/retos.v41i0.78201

Pegalajar, M. (2017). An analysis of self-concept in students with compensatory education needs for developing a mindfulness-based psychoeducational program. SAGE Open, 7(2), 2158244017708818. https://doi.org/ $10.1177 \% 2 F 2158244017708818$

Simón, C., \& Giné, C. (2016). Escuela, familia y comunidad: construyendo alianzas para promover la inclusión. Revista latinoamericana de educación inclusiva, 10(1), 25-42. http: / / dx.doi.org/10.4067/S071873782016000100003

Shulman, L. S. (2005). Conocimiento y enseñanza: fundamentos de la nueva reforma. Profesorado, Revista de curriculum y formación del profesorado, 9(2), 1-30. https: / / revistaseug.ugr.es/index.php/profesorado/article/ view/19743/19229

Soto, R. (2003). La inclusión educativa: Una tarea que le compete a toda una sociedad. Actualidades investigativas en educación, 3(1). https: / /www.redalyc.org/pdf/447/ 44730104.pdf

Tenorio, S. (2005). La integración escolar en Chile: Perspectiva de los docentes sobre su implementación. REICE. Revista Iberoamericana sobre Calidad, eficacia y cambio en educación, 3(1), 823-831. https://www.redalyc.org/pdf/ 551/55130176.pdf

UCE (2021). Educación Física y Salud. https:// www.curriculumnacional.cl/portal/Educacion-Gene$\mathrm{ral} /$ Educacion-fisica-y-salud/

UNICEF (2005). Seminario Internacional: Inclusión social, discapacidad y políticas públicas. https: / / www.unicef.cl/ a r c hivos_d o c u m e n t o / $200 /$ Libro\%20seminario\%20internacional\%20discapacidad.pdf Vila, I., Avendaño, R., Linzmayer, L., Mora, M., Duarte, E., \& Pacheco, J. (2021). Inclusión de estudiantes con discapacidad visual en clases de Educación Física. Revista Horizonte Ciencias De LaActividad Física, 11(2), 1-13. http: / /www.revistahorizonte.ulagos.cl/index.php/horizonte/article/view/183

Villalobos, M. (2015). La Familia: Formadora Primigenia. Perspectiva Educacional, Formación de Profesores, 54(2), 3-19. https: / / www.redalyc.org/pdf/3333/ 333339872002.pdf

Warnock, M. (1987). Encuentro sobre necesidades de educación especial. Revista de educación. https:// dialnet.unirioja.es/ ejemplar/1977 